\title{
Evaluation of Disease Patterns, Treatment and Prognosis of Tuberculosis in AIDS Patient
}

\author{
Angela Naomi Atomiya, David Emerson Uip \\ and Olavo Henrique Munhoz Leite
} Casa da AIDS Outpatient Clinic, São Paulo,
SP, Brazil

\begin{abstract}
Patterns of disease, diagnosis, treatment and prognosis of tuberculosis in 100 patients coinfected with AIDS at Casa da AIDS clinic was studied. Demographic characteristics were as follows: 76 male patients, 24 female patients, 67 caucasian, average 35.8 years-old (SD \pm 8.5). Sexual transmission of HIV was reported in 68 patients. Pulmonary tuberculosis was seen in 40 patients, extrapulmonary in 11, and combined in 49 patients. In 63 patients, TCD $_{4}^{+}$ counts were below $200 / \mathrm{mm}^{3}$ when tuberculosis was diagnosed. Fifty-five patients had their diagnoses confirmed by bacteriological identification of Mycobacterium; either through direct observation and/or culture. Tuberculosis was treated with rifampin, isoniazid and pyrazinamide in 60 patients, reinforced treatment in 14 and alternative treatment in the other 13 patients. Tuberculosis therapy lasted up to 9 months in $66 \%$ of the patients. Fiftyfour patients were treated with a two-drug antiretroviral regimen and the remaining 46 patients received a triple regimen, which included a protease inhibitor. Among the latter, 35 patients were co-treated with rifampin. The occurrence of hepatic liver enzyme abnormalities was statistically related to alternative antiretroviral regimens $(p=0.01)$ and to the coadministration of rifampin and protease inhibitor $(p=0.019)$. Clinical resolution of tuberculosis was obtained in 74 patients. Twelve patients died during tuberculosis treatment. Resolution of tuberculosis was statistically significant related to antituberculosis treatment adherence $(p=0.001)$. The risk of no response to the treatment was 1.84 times more frequent among patients treated with alternative regimens regardless of the duration of the therapy. We conclude that the characteristics of tuberculosis in HIV infected patients requires that special attention be directed to the types and duration of both antiretroviral and anti-TB therapy in order to achieve the highest level of care.
\end{abstract}

Key Words: AIDS, tuberculosis, rifampin, isoniazid, pyrazinamide.

There are about 8 million cases of tuberculosis (TB) every year, resulting in 2 million deaths worldwide [1]. In the U. S., TB cases were declining by about $5 \%$ annually until 1985, when, TB emerged as one of the most important complications of human immunodeficiency virus (HIV) infection [2]. Since the

Received on 23 May 2001; revised 23 November 2001.

Address for correspondence: Dr. Angela Naomi Atomiya. Casa da AIDS Outpatient Clinic. R. Frei Caneca, 557 São Paulo/SP Zip code: 01307-001. Phone/Fax: 011) 256-7562.

E-mail: atomiya@bol.com.br

The Brazilian Journal of Infectious Diseases 2002;6(1):29-39 (C) 2002 by The Brazilian Journal of Infectious Diseases and Contexto Publishing. All rights reserved.

$1413-8670$ beginning of the acquired immunodeficiency syndrome (AIDS) epidemic, more than 47 million people in the world have been infected by HIV [3]. In 2000, it was estimated that about $14 \%$ of active TB occurrences were related to HIV [4].

\section{Objective}

The purpose of this study was to conduct a case series review of symptom based clinical diagnoses, laboratory findings, and treatment outcomes of TB patients co-infected with HIV who were treated at the Casa da AIDS outpatient clinic in São Paulo. 


\section{Material and Methods}

Between January 1, 1996, and December 31, 1997, the Epidemiology Service of São Paulo University Clinical Hospital was notified of 742 cases of tuberculosis and 642 cases of AIDS. From these cases, 100 patients were identified as being co-infected with both TB and AIDS, and therefore suitable for this study. The medical records of the patients were evaluated retrospectively. In this study, TB is categorized as: pulmonary TB, extrapulmonary TB, and combined TB which includes both pulmonary and extrapulmonary. An antiretroviral regimen (use of protease inhibitor or not) and TB therapy (plan 1: rifampin, isoniazid and pyrazinamide; plan 1R: rifampin, isoniazid, pyrazinamide, and ethambutol; and an alternative plan) were also evaluated.

Statistical analysis. Descriptive, univariate, multivariate, and stratified analyses were carried out using the STATA program version 6.0. A Chi Square test was used to evaluate differences in proportions. The Student t-test was used for mean comparisons. Comparisons at $\mathrm{p}<0.05$ were considered significant.

\section{Results}

Patient demographics are listed in Table 1. HIV distribution and routes of exposure are listed according to gender in Table 2.

Previous opportunistic infections were reported at the time of the TB diagnosis in 31 patients (31\%). Nine patients (9\%) had been previously treated for tuberculosis, and five patients (5\%) indicated that they had come in contact with TB by exposure to infected person in their home.

At the time of HIV infection, 47 patients (47\%) had been recently diagnosed with tuberculosis (within 3 months) and the remaining 53 patients (53\%) were diagnosed with TB while being treated for HIV (between 4 and 132 months).

The diagnosis of tuberculosis was based on clinical diagnosis plus at least one other complementary exam (Table 3).
The most frequent clinical presentation was combined TB (49\%) followed by pulmonary TB (40\%) and extrapulmonary TB (11\%).

The clinical manifestations varied, and are listed in Table 4 according to the tuberculosis presentation.

No significant difference was observed between the type of TB found according to gender $(\mathrm{p}=0.20)$, age group $(\mathrm{p}=0.49)$, category of HIV exposure $(\mathrm{p}=0.51)$, or $\mathrm{TCD}_{4}^{+}$lymphocyte counts at the beginning of the specific treatment $(\mathrm{p}=0.32)$. Sixty-three $(63 \%)$ patients had $\mathrm{TCD}_{4}{ }^{+}$lymphocyte counts $<200$ cells $/ \mathrm{mm}^{3}$ at the start of the TB treatment, and 24 (24\%) of these patients had counts $\leq 50$ cells $/ \mathrm{mm}^{3}$.

Radiological abnormalities were observed in $97 \%$ of the patients with pulmonary $\mathrm{TB}$, and in $100 \%$ of the patients with combined TB. One patient with pulmonary tuberculosis showed a normal chest X-ray result; however, sputum samples and cultures were positive for acid-fast bacilli (AFB).

Among the radiological findings, pulmonary infiltrate was the main abnormality, occurring in $84.4 \%$ of the patients with pulmonary TB, and in $78.3 \%$ with combined TB. Cavitation was observed in $6.3 \%$ and $6.5 \%$ of the pulmonary and combined presentations, respectively.

The demonstration of the etiological agent by direct observation (positive and non-conducted) and by culture (positive and non-conducted) was most frequent among the patients with combined TB (Table 5). The demonstration of the etiological agent was more frequent in sputum samples (34\%) than in lymph node biopsies (16\%) (Table 6).

Image-diagnosis exams were conducted in 6 extrapulmonary TB patients (54.5\%), and in 26 combined TB (53.1\%) patients. The results are presented in Table 7.

Pathological analysis of tissues was conducted on 6 extrapulmonary TB patients (54\%) and 28 combined TB (57.1\%) patients. Biopsies from 3 extrapulmonary TB patients (50\%) showed granuloma. Caseating necrosis was observed in 1 of these 3 patients (16.9\%). In the patients with combined TB, $23(82.1 \%)$ patients demonstrated granuloma, and 12 (42.9\%) patients had caseating necrosis. In 9 combined TB (32.1\%) patients, granuloma and caseating necrosis were found. 
Table 1. Demographics

\begin{tabular}{lc}
\hline Variable & $\mathbf{N}(\%)$ \\
\hline $\begin{array}{l}\text { Gender } \\
\text { Female } \\
\quad \text { Male }\end{array}$ & $24(24 \%)$ \\
Race & $76(76 \%)$ \\
$\quad$ Caucasian & $67(67 \%)$ \\
$\quad$ Mulatto & $11(11 \%)$ \\
$\quad$ American African & $22(22 \%)$ \\
Age group & \\
20 to 29 years & $26(26 \%)$ \\
30 to 39 years & $45(45 \%)$ \\
$\geq 40$ years & $29(29 \%)$ \\
Total & $100(100 \%)$ \\
\hline
\end{tabular}

Table 2. Patient distribution by HIV category of exposure according to gender

\begin{tabular}{lcc}
\hline Category of exposure* & $\begin{array}{c}\text { Female } \\
\mathbf{n}(\%)\end{array}$ & $\begin{array}{c}\text { Male } \\
\mathbf{n}(\%)\end{array}$ \\
\hline Sexual Practice & & \\
$\quad$ Heterosexual & $18(75 \%)$ & $24(32 \%)$ \\
$\quad$ Homosexual & - & $24(32 \%)$ \\
$\quad$ Bisexual & - & $2(3 \%)$ \\
Blood & - & $17(22 \%)$ \\
$\quad$ IV drug use & $2(8 \%)$ & $1(1 \%)$ \\
$\quad$ Blood transfusion & $4(17 \%)$ & $8(10 \%)$ \\
Unknown & $24(100 \%)$ & $76(100 \%)$ \\
Total &
\end{tabular}

*More than one category of exposure in 4 patients. 
Table 3. Diagnosis form of the tuberculosis

\begin{tabular}{lc}
\hline Complementary exams & Number of patients n (\%) \\
\hline $\begin{array}{l}\text { Identification of the agent } \\
\text { (Direct observation and/or culture) } \\
\text { Yes }\end{array}$ & $55(55 \%)$ \\
No & $45(45 \%)$ \\
Nes & \\
(granuloma and/or caseating necrosis)* & $31(31 \%)$ \\
No & $67(67 \%)$ \\
$\begin{array}{l}\text { Suggestive radiological alteration** } \\
\text { (pleural effusion, cavitation, miliary infiltrate) } \\
\text { Yes }\end{array}$ & $37(37 \%)$ \\
No & $46(46 \%)$ \\
\hline
\end{tabular}

*shows 2 ignored amounts.

**shows 7 ignored amounts.

Table 4. Clinical manifestations according to the tuberculosis presentation

\begin{tabular}{lccc}
\hline $\begin{array}{l}\text { Clinical } \\
\text { manifestations }\end{array}$ & $\begin{array}{c}\text { Pulmonary* } \\
\mathbf{n}(\%)\end{array}$ & $\begin{array}{c}\text { Extrapulmonary } \\
\mathbf{n}(\%)\end{array}$ & $\begin{array}{c}\text { Combined*** } \\
\mathbf{n}(\%)\end{array}$ \\
\hline Fever & $33(82.5 \%)$ & $6(54.6 \%)$ & $41(83.7 \%)$ \\
Cough & $30(75.0 \%)$ & - & $32(65.3 \%)$ \\
Sweating & $7(17.5 \%)$ & $1(9.1 \%)$ & $12(24.5 \%)$ \\
Chills & $3(7.5 \%)$ & - & $1(2.0 \%)$ \\
Weight Loss & $12(30.0 \%)$ & $2(18.2 \%)$ & $25(51.0 \%)$ \\
Lymphadenopathy & $2(5.0 \%)$ & $4(36.4 \%)$ & $22(44.9 \%)$ \\
Asthenia & $6(15.0 \%)$ & - & $6(12.2 \%)$ \\
Other & $22(55.0 \%)$ & $8(72.8 \%)$ & $19(38.8 \%)$ \\
\hline
\end{tabular}

*No information on 3 patients.

**No information on 1 patient. 
Table 5. Demonstration of the etiological agent according to tuberculosis presentation

\begin{tabular}{lccc}
\hline Examination & $\begin{array}{c}\text { Pulmonary TB } \\
\mathbf{n = 4 0} \\
\mathbf{n}(\boldsymbol{\%})\end{array}$ & $\begin{array}{c}\text { Extrapulmonary TB } \\
\mathbf{n = 1 1} \\
\mathbf{n}(\boldsymbol{\%})\end{array}$ & $\begin{array}{c}\text { Combined TB } \\
\mathbf{n = 4 9} \\
\mathbf{n}(\boldsymbol{\%})\end{array}$ \\
\hline $\begin{array}{l}\text { Positive direct } \\
\text { observation }\end{array}$ & $20(50.0 \%)$ & $1(9.1 \%)$ & $27(55.1 \%)$ \\
$\begin{array}{l}\text { Non-conducted } \\
\text { direct observation }\end{array}$ & - & $5(45.5 \%)$ & $6(12.2 \%)$ \\
$\begin{array}{l}\text { Positive culture } \\
\text { Non-conducted } \\
\text { culture }\end{array}$ & $11(27.5 \%)$ & - & $21(42.9 \%)$ \\
\hline
\end{tabular}

Table 6. Demonstration of the etiological agent according to the clinical tuberculosis presentation

\begin{tabular}{lccc}
\hline Local & $\begin{array}{c}\text { Pulmonary TB } \\
\mathbf{n = 4 0}\end{array}$ & $\begin{array}{c}\text { Extrapulmonary TB } \\
\mathbf{n = 1 1}\end{array}$ & $\begin{array}{c}\text { Combined *TB } \\
\mathbf{n = 4 9}\end{array}$ \\
\hline Sputum & $20(50.0 \%)$ & - & $14(28.6 \%)$ \\
Blood & - & - & $2(4.1 \%)$ \\
Bronchoalveolar lavage fluid & - & - & $3(6.1 \%)$ \\
Pleural Liquid & - & - & $1(2.0 \%)$ \\
Bone marrow biopsy & - & - & $1(2.0 \%)$ \\
Biopsy materials & & - & $1(2.0 \%)$ \\
$\quad \begin{array}{l}\text { lung } \\
\quad \text { lymph nodes }\end{array}$ & & $1(9.1 \%)$ & $15(30.6 \%)$ \\
$\quad$ liver & & - & $1(2.0 \%)$ \\
$\quad$ pericardium & & - & $1(2.0 \%)$ \\
$\quad$ pleura & & - & $1(2.0 \%)$ \\
\hline
\end{tabular}

*The etiological agent was demonstrated in more than one local in 6 patients. 
Table 7. Radiological findings according to the clinical form of the tuberculosis

\begin{tabular}{lccc}
\hline $\begin{array}{l}\text { Ultrasound findings } \\
\text { and/or computerized } \\
\text { tomography }\end{array}$ & $\begin{array}{c}\text { Extrapulmonary TB } \\
\mathbf{n}(\%)\end{array}$ & $\begin{array}{c}\text { Combined TB } \\
\mathbf{n}(\%)\end{array}$ & $\begin{array}{c}\text { Pulmonary TB } \\
\mathbf{n}(\%)\end{array}$ \\
\hline Hepatomegaly & - & $20(40.8 \%)$ & $5(12.5 \%)$ \\
Splenomegaly & $1(9.1 \%)$ & $16(32.7 \%)$ & $6(15.0 \%)$ \\
Lymphadenopathy & $2(18.2 \%)$ & $17(34.7 \%)$ & $2(5.0 \%)$ \\
Lymphadenopathy & $1(9.1 \%)$ & $4(8.2 \%)$ & $1(2.5 \%)$ \\
with liquefaction & $1(9.1 \%)$ & $5(10.2 \%)$ & - \\
Ascites & - & $10(20.4 \%)$ & - \\
Pleural effusion & - & $3(6.1 \%)$ & - \\
Pericardial effusion & $3(27.3 \%)$ & - & - \\
Other & & & \\
\hline
\end{tabular}

Table 8. Distribution of the tuberculosis of the treatment plans

\begin{tabular}{lc}
\hline Tuberculosis treatment plan & Frequency n(\%) \\
\hline$* E 1$ & $60(60 \%)$ \\
$* *$ E1R & $14(14 \%)$ \\
Alternative plan & $26(26 \%)$ \\
Total & $100(100 \%)$ \\
\hline
\end{tabular}

*Plan 1 (rifampin, isoniazid, pyrazinamide).

** Plan 1R (rifampin, isoniazid, pyrazinamide, etambutol).

Table 9. Distribution of the patients according to the duration of the therapy

\begin{tabular}{lc}
\hline Tuberculosis treatment time & $\begin{array}{c}\text { Frequency } \\
\mathrm{n}(\%)\end{array}$ \\
\hline$\leq 6$ months & $20(20 \%)$ \\
7 to 9 months & $46(46 \%)$ \\
10 to 12 months & $21(21 \%)$ \\
$\geq 13$ months & $13(13 \%)$ \\
Total & $100(100 \%)$ \\
\hline
\end{tabular}


Evolutionary aspects. Initially, $75 \%$ of the patients with tuberculosis were treated with rifampin, isoniazid, and pyrazinamide (treatment plan 1). However, 24 (24\%) patients changed their treatment plans in the course of the treatment (Table 8). Three patients had plans for the TB treatment containing rifampin $(\mathrm{R} / \mathrm{H} / \mathrm{E}$, $\mathrm{R} / \mathrm{H} / \mathrm{E} / \mathrm{CFX}, \mathrm{R} / \mathrm{H} / \mathrm{Z} / \mathrm{S}$ respectively) however distinct from $\mathrm{E} 1$ and $\mathrm{E} 1 \mathrm{R}$. These were considered as alternative schemes in the TB schemes distribution analysis.

The duration of tuberculosis therapy was 7 and 9 months in $46(46 \%)$ of the patients and longer than 9 months in 34 (34\%) patients (Table 9).

Hepatic liver enzymes. An increase of hepatic liver enzymes over normal limits occurred in 49 (49\%) patients. Of these, 23 (47\%) demonstrated increases up to 5 times the norm. Significant differences were observed in hepatic liver enzyme alterations in $45 \%$ of patients who had alternative treatment plans for the tuberculosis $(p=0.01)$. In $46 \%$ of these cases, the increases were up to 5 times the normal value $(\mathrm{p}=$ 0.009).

Antiretroviral therapy. Similar to the TB treatment plans, the antiretroviral therapies were modified during the course of the treatment. Most of the patients (72\%) used non-protease inhibitor therapies. Nevertheless, $49 \%$ of treatment plan swaps were observed, which considered whether or not patients were evaluated using the protease inhibitor (PI), during the antituberculosis therapy.

Forty six (46\%) patients were treated with PI, and $27(59 \%)$ of these patients maintained their treatment plans after the TB diagnosis. It was also observed that rifampin and PI were co-administered to 35 (76\%) patients (15 indinavir, 13 saquinavir and 7 ritonavir).

There was a significant relationship $(p=0.019)$ between alterations of hepatic liver enzymes and the use of rifampin plus a PI. However, this relationship was not significant in cases where hepatic liver enzymes were increased to 5 times the normal value $(p=0.167)$.

Duration of TB therapy. Cure rates were found to be statistically related to treatment duration $(\mathrm{p}=0.001)$.
Only $8.1 \%$ of treated patients were cured after 6 months of therapy. The cure rate jumped to $51.4 \%$ if treatment lasted 7 to 9 months.

Duration of TB therapy was also significantly linked to TB presentation $(p=0.003)$. Among patients who were treated for 6 months, or 7 to 9 months, the pulmonary presentation prevailed; whereas with longer treatments of 12 months or more, combined TB prevailed.

Cure. Seventy four (74\%) patients had their TB cured. Two (2\%) patients were transferred to other services and recovery information could not be obtained. There was a significant relationship ( $\mathrm{p}=$ 0.01) between the cure of TB and maintaining TB treatment. Eighteen (18\%) patients abandoned TB treatment. Fourteen patients became ineligible for clinical assistance and, in 4 patients, the cure of the TB was achieved after re-treating the patients. No relationship between cure according to PI use ( $\mathrm{p}=$ $0.7)$ and TB treatment plan ( $\mathrm{p}=0.1)$ was observed. No significant differences were observed $(p=0.7)$ between curing TB and treatment group (patients who showed PI-rifampin interaction, patients with PI use with no interaction, patients with no PI use but with treatment plans containing rifampin, and patients not using PI and rifampin).

Deaths. New opportunistic infections occurred in 18 (18\%) patients during TB treatment. During the specific therapy, $9(9 \%)$ patients died as a result of TB. Necropsy data showed that deaths resulted as follows: 2 patients died from tuberculous meningitis, 1 patient died from pulmonary TB, 2 patients died from combined TB with CNS involvement, and 4 patients died from combined TB and other site involvement. Three patients died from other opportunistic infections. Necropsy revealed 2 deaths resulting from Kaposi's sarcoma; 1 was a bacterial pneumonia and 1 was disseminated neurocryptococcosis. TB deaths were not correlated with $\mathrm{TCD}_{4}^{+}$lymphocyte counts either at the beginning or at the end of the TB treatment $(\mathrm{p}=0.8$ and $\mathrm{p}=$ 0.3 , respectively). Deaths were not significantly 
related to PI usage during treatment of the $\mathrm{TB}(\mathrm{p}=$ $0.6)$, TB treatment plan $(\mathrm{p}=0.3)$, duration of TB therapy $(p=0.3)$, or the presence of previous or new opportunistic infections $(\mathrm{p}=0.3$ and $\mathrm{p}=0.09$, respectively).

TB treatment results were obtained in 98 (98\%) cases (cure or non-cure). Twenty-four of these were not cured after the applied treatment; therefore, in the study sample, the prevalence of non-cure was $24.5 \%$ $($ CI $95 \%=15.8-33.2)$.

\section{Discussion}

Infection with human immunodeficiency virus exerts an important influence in the pathogenesis and natural history of TB [5]. Although tuberculosis can attack individuals at any stage of HIV infection, the reactivity to Purified Protein Derivative (PPD) and the clinical and radiological manifestations vary considerably depending on the severity of the immunodepression [6-10].

It was observed in this study that, with the predominance of heterosexual transmission of HIV among young adults, the proportion AIDS-TB coinfection is distributed in a ratio of 3 men to 1 woman, although this tendency has been changing over the last few years. Apart from gender-distribution, the HIV epidemic also reflects socioeconomic and demographic factors. HIV affects both poor and rich, and is spreading to small cities and towns [11].

M. tuberculosis is considered more virulent than other microorganisms, perhaps because of its earlier appearance and its more frequent association with AIDS [12,13]. The diagnosis of TB can occur concomitantly to the diagnosis of the HIV infection. In our study, both situations were observed. In studies of patients prior to the HIV epidemic, approximately $85 \%$ of the TB cases were restricted to the lungs and the remaining $15 \%$ showed combined or exclusively extrapulmonary involvement [14]. After the start of the HIV epidemic, however, this distribution has been changed [15]. In this study, it was observed that $40 \%$ of TB cases were pulmonary, $11 \%$ were extrapulmonary, and $49 \%$ were combined.
In 1997, Alpert et al., reported that New York HIV infection patients had the following types of TB: $43.5 \%$ $(n=40)$ of TB cases were pulmonary, $21.7 \%(n=20)$ were extrapulmonary, and $34.8 \%(n=32)$ were combined [16].

In this study, the majority $(63 \%)$ of the patients showed severe immunodepression at the beginning of TB treatment with $\mathrm{TCD}_{4}^{+}$lymphocyte counts being < 200 cells $/ \mathrm{mm}^{3}$ which did not allow a correlation to be made between different TB presentations and immunodepression levels.

The signs and symptoms reported by the patients were also non-specific. The signs and symptoms were similar to other opportunistic diseases and could not be used to specifically make a diagnosis of TB [17].

Among radiological findings, pulmonary infiltrate was the main abnormality. Lung cavitation was observed in only a small number of patients with combined and pulmonary TB presentation, and, in 1 patient with pulmonary TB the X-ray was considered normal.

In 72 patients with pulmonary TB and HIV infection, Alpert et al., noted the following radiological findings: $53 \%$ had focal pulmonary infiltration, $26 \%$ had infiltrate in superior lobes, $7 \%$ had cavitations, 39\% had tuberculous imediastinal lymph nodes, and $11 \%$ had normal thorax radiography [16].

Bacteriological confirmation of Mycobacterium was obtained in only $55(55 \%)$ patients through direct exam and/or culture. However, a positive culture, with no species identification, does not necessarily imply causality. Patients with $\mathrm{TCD}_{4}^{+}$lymphocyte counts of $<$ 50 cells $/ \mathrm{mm}^{3}$ may have had a mycobacteria nontuberculosis infection (MNT).

In 1998, Afessa et al., published the result obtained after 12 years of autopsy observations and described the pulmonary complications in HIV infected patients. These authors showed that $33 \%$ of pulmonary infections were by mycobacterias and most of them were considered to be non- tuberculosis [18].

Despite these considerations, a significant relationship to TB cure has not been found based upon the method of diagnosis, whether by X-ray, or identification through direct exam, or based on culture or pathological exam. 
Diagnosis is also a challenge in clinical practice. Challenges include patient flow, adequate systematization and the availability of health resources. The orientation of a patient's examination is also critical. It is our concern that diagnosed cases with no etiologic agent recovery do not allow the correct diagnosis and result in unnecessary or inadequate treatment.

Results obtained in our study reveal an improvement in TB treatment outcomes in patients infected by HIV/AIDS compared to previous studies.

Dupon AND Ragnaud reported that, of 123 patients, TB was cured in $57.7 \%$ of the patients and $26.8 \%$ of the patients died while being treated for an AIDS and TB co-infection. Among the deaths, 6.5\% were caused by TB [19].

In our study, $74 \%$ of patients were cured and $18 \%$ abandoned treatment. TB resulted in $9 \%$ of the patients dying. In $3 \%$ of the patients, deaths were caused by other opportunistic infections.

The cure of TB was associated with adherence to the TB treatment and its duration. During the development of streptomycin (1944), TB therapy was 24 months long; it has been gradually reduced to a 6 month regimen [20].

Along with AIDS, the standard treatment for TB has been extended to 9 months due to poor immune response and high rates of relapse. However, the duration for HIV-infected patients has been recently revised. Six month therapy and supervised treatment plans 2 or 3 times a week for patients with HIV/AIDS have been made possible with powerful antiretroviral therapy for an immune recovery of the patients [21,22].

Despite these findings, the highest rate of TB cure occurred among the patients with 7 to 9 months of treatment $(51.4 \%$ patients were cured when the TB therapy was conducted from 7 to 9 months, compared to $8.1 \%$ with 6 month therapy).

As well as investigating the duration of therapy, the composition of the anti-TB regimen was evaluated. According to the literature, the available treatments which include rifampin show a therapeutic success rate from $90 \%$ to $95 \%$ [22].
The univariated analysis showed a tendency of lower cure frequency for those patients who used alternative treatment plans without rifampin, although without achieving a significance $(\mathrm{p}=0.10)$.

It was observed that the risk, of non-cure of an individual who used the alternative method of TB treatment, whatever the time, was 1.84 times greater than the risk of non-cure of a patient who received treatment plan 1 (rifampin, isoniazid and pyrazinamide).

In general, anti-TB treatment plans are prescribed based on HIV infection severity $\left(\mathrm{TCD}_{4}^{+}\right.$lymphocyte counts) and the clinical presentation of the TB. Alternative regimens, with no rifampin inclusion, are used over a 12 month period [22].

Unexpectedly, TB cure rates showed an inverse relationship to the use of the protease inhibitors in the antiretroviral regimen. It is known that adherence to the therapeutic regimen is essential for obtaining a reasonable response with anti-infectious therapy and to prevent the emergence of resistance, so the alternative treatment plans may have become obstacles to cure. This may be the result of the use of multiple drugs, complex regimens, and the toxicity of the medications.

A significant relationship was not found among noncure and clinical presentation and hepatic liver enzyme alterations during treatment.

A factor that may have interfered with therapeutic success is the metabolic interaction between antituberculosis therapy and antiretrovirals. The concomitant use of rifampin and protease inhibitors was observed in $35 \%$ of these patients. Other authors also reported this interaction between the protease inhibitors and the rifampin. Between July, 1996, and August, 1998, at least 65 (63\%) out of 104 HIV-infected patients with TB received concomitant therapy of PI and rifampin in New York [23].

It was observed that, by the end of the antituberculous therapy there was no significant differences in the immune response (evaluated by $\mathrm{TCD}_{4}^{+}$lymphocyte counts) between antiretroviral therapies (to include or not include protease inhibitors) and TB regimens. 
Patients who used rifampin may have had an interaction with PI therapy, which may have been related to inadequate suppression of the HIV and to a poor anti-retroviral response [24].

\section{Conclusion}

Patients co-infected with AIDS and TB showed a high frequency of extrapulmonary and dissemminated forms, as well as a prevailing severe immunodepression. $\mathrm{TCD}_{4}^{+}$lymphocytes counts were $<200$ cells $/ \mathrm{mm}^{3}$ in the different presentations of the TB.

Unspecific clinical manifestations and identification of the etiologic agent (by direct exam and/or culture) in little more than half of the patients, require that clinical diagnostic methods must be examined.

Early and late complications of tuberculosis resulted in mortality. New opportunistic infections led to immune deterioration that may have been induced by tuberculosis.

Classical therapy with rifampin, isoniazid, and pyrazinamide and the patient adherence to the tuberculosis treatment were factors associated with reasonable evolution of the disease.

Although antiretroviral therapy influences a host response to the tuberculosis organism, in this study, a better evolution of the disease was not observed in patients who used triple antiretroviral therapy, including the protease inhibitor, compared to patients on other therapeutic regimens.

Co-administration of rifampin and protease inhibitors did not affect the evolution of the TB clinical cure, but was associated with the occurrence of increases in hepatic liver enzymes.

TB control depends on resolution at all stages of the disease, from the initial suspicion of TB until the end of the therapy. The data obtained in this study must be studied carefully in order to direct future studies to determine the best strategy to control AIDS and TB co-infection.

\section{Acknowledgements}

The authors are very grateful to Robert James Hughes for reviewing this manuscript, Patricia Braga and Maria de Fátima Marinho de Souza for technical assistance in the statistical analysis.

\section{References}

1. World Health Organization (WHO). Tuberculosis. Disponível em: <http:// www.who.int/inf-fs/en/ fact104.html >. April, 2000.

2. Centers for Disease Control and Prevention. Tuberculosis morbidity - United States, 1992. MMWR Morb Mort Wkly Rep 1993;42(n.RR-36):696-7, 703-4.

3. World Health Organization (WHO). HIV/AIDS epidemic surveillance. Report on the Global HIV/AIDS epidemic. June 2000.

4. Chaisson R.E. Tuberculosis. Disponível em: <http:// hopkins-id.edu/diseases/tb/tb.html>. January, 1999.

5. Selwyn P.A., Hartel D., Lewis V.A., et al. A prospective study of the risk of tuberculosis among intravenous drug users with human immunodeficiency virus infection. N Engl J Med 1989;320:545-50.

6. Barnes P.F., Bloch A.B., Davidson P.T., Snider D.E. Tuberculosis in patients with human immunodeficiency virus infection. N Engl J Med 1991;324:1644-50.

7. Chaisson R.E., Slutkin G. Tuberculosis and human immunodeficiency virus infection. J Infect Dis 1989; 159:96-100.

8. Fonseca L.S., Kritsky A., SantÁnna C., et al. Relationship between immune response and clinical manifestations in patients with tuberculosis. Braz J Med Biol Res 1992;25:49-52.

9. Havlir D.V., Barnes P.F. Tuberculosis in patients with human immunodeficiency virus infection. NEngl J Med 1999;340:367-73.

10. Shafer R.W., Edlin B.R. Tuberculosis in patients infected with human immunodeficiency virus: perspective on the past decade. Clin Infect Dis 1996;22:683-704.

11. Dhalia C., Barreira D., Castilho E.A. A AIDS no Brasil: situação atual e tendências. AIDS: Bol Epidemiol, v.13, n.1, p.3-51, dez. 1999/ jun. 2000.

12. Mukadi Y., Perriëns J.H., St. Louis M.E., et al. Spectrum of immunodeficiency in HIV-1 infected patients with pulmonary tuberculosis in Zaire. Lancet 1993; 342:143-6.

13. Theuer C.P., Hopewell P.C., Elias D., et al. Human immunodeficiency virus infection in tuberculosis patients. J Infect Dis 1990;162:8-12. 
14. Farer L.S., Lowell L.M., Meador M.P. Extrapulmonary tuberculosis in the United States. Am J Epidemiol 1979;109:205-17.

15. Silva L.C.C. Tuberculose extrapulmonar (excetuando o derrame pleural). J Pneumol 1993;19:83-7.

16. Alpert P.L., Munsiff S.S., Gourevitch M.N., et al. A prospective study of tuberculosis and human immunodeficiency virus infection: clinical manifestations and factors associated with survival. Clin Infect Dis 1997;24:661-8.

17. Griffith D.E. Mycobacteria as pathogens of respiratory infection. Infect Dis Clin North Am 1998;12:593-611.

18. Afessa B., Green W., Chiao J., Frederick W. Pulmonary complications of HIV infection. Chest 1998; $113: 1225-9$.

19. Dupon M., Ragnaud J.M. Tuberculosis in patients infected with human immunodeficiency virus 1 . A retrospective multicentre study of 123 cases in France. Q J Med 1992;85:719-30.

20. Ellner J.J., Hinman A.R., Dooley S.W., et al. Tuberculosis Symposium: emerging problems and promise. J Infect Dis 1993; 168:537-51.

21. Centers for Disease Control and Prevention. Prevention and treatment of TB among patients infected with HIV: principles and therapy and revised recommendations. MMWR Morb Mort Wkly Rep 1998;47(n.RR-20):1-58.

22. Ministério da Saúde. Recomendações para terapia antiretroviral em adultos e adolescentes infectados pelo HIV, 2000. <http://www.aids.gov.br/ consensonovo.pdf $>$. November, 2000.

23. Sundaram V., Driver C.R., Munsiff S.S. Tuberculosis treatment practices in the era of protease inhibitors: a provider survey. AIDS 1999; 13:149-50.

24. Flexner C. HIV-protease inhibitors. N Engl J Med 1998;338:1281-92. 